\title{
Evaluation of Local Radii of Atom-Probe-Tomography Specimens
}

\author{
S.S.A. Gerstl, B.P. Geiser, T.F. Kelly and D.J. Larson
}

Imago Scientific Instruments Corp., 5500 Nobel Drive, Madison, WI 53711-4951

In atom probe tomography (APT) [1], ions are field evaporated from the apex of a sharp needleshaped specimen and detected on a position sensitive detector. Subsequently the data are reconstructed in a computer, enabling researchers to image a small volume (typically 100 by 100 by $100 \mathrm{~nm}$ ) of a specimen in three dimensions on the nanometer scale. During the field evaporation process, the specimen apex acquires a steady-state endform (for a single phase material). Theoretically for a sphere of material in free space, this equilibrated shape (neglecting crystallographically dependent work functions) is spherical with a radius approximately $\mathrm{R}=\mathrm{V} / \mathrm{kF}$, where $\mathrm{V}$ is the voltage applied to the specimen, $\mathrm{F}$ is the material-dependent evaporation field [1], and $k=1$. For a needle geometry, $k>1[2,3]$ and the needle endform is known to assume a nonhemispherical shape. This makes it challenging to achieve accurate reconstructions since commonly employed reconstruction algorithms assume a spherical endform [4]. In order to improve the accuracy of APT data reconstruction, it is advantageous to make a more accurate measurement of the average radius of a specimen than that obtained from a spherical fit to the apex of the needle. An analysis tool has been developed to measure the variation of local radii of APT specimen endforms imaged via HR-SEM or TEM.

The algorithm enables the fitting of a polynomial equation (of user-selectable order) to electron microscopy images of an atom probe specimen (Fig. 1a), measuring its radius along the region of the dark line in Fig. 1b. The comparison between a best-fit circle (dashed) to a $5^{\text {th }}$ order polynomial fit is shown in Fig. 1c. This local radius curve reveals the smaller local radii (minima) near the evaporated edges of the endform and larger local radii (maxima) near the apex. The ratio of maxima to the mean of the minima is used here to estimate the ellipticity (ellipticity $=1$ for a sphere), which has been explored previously in field ion microscopy analysis of tip shapes [5]. Ellipticity can be measured as a function of various analysis parameters. The local radius curves of three evaporated Si tips [6] shown in Fig. 2(a-c) are overlaid in Fig. 3, revealing a decreasing trend in ellipticity with increasing bluntness, Fig. 4 (trendline is logarithmic).

The details revealed by local radii measurements, particularly the degree of deviation from hemispherical, can aid in choosing an average radius (Fig. 1c) for more accurate reconstructions. The extension of the measurement method to other materials and to more complex tip shapes will be presented.

[1] M. K. Miller et al. (1996). Atom Probe Field Ion Microscopy. Oxford, Oxford University Press.

[2] R. Gomer, (1965) Field Emission and Field Ionization, New York, American Institute of Physics, p. 32.

[3] D. J. Larson K. F. Russell and M. K. Miller, Microsc. Microanal. 5 (1999) 930.

[4] P. Bas, et al., Appl. Surf. Sci. 87/88 (1995) 298.

[5] Elswijk, H. B et al., J. de Phys. 49(11): C6-489 (1988) 493.

[6] A. Shariq, et al., Ultramicroscopy (2008), doi:10.1016/j.ultramic.2008.10.001 


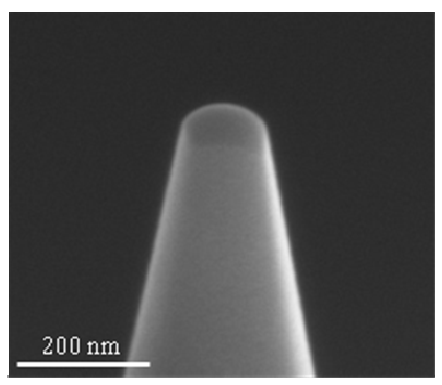

(a)

Figure 1

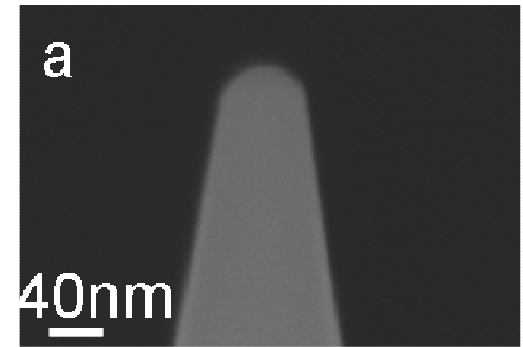

Figure 2

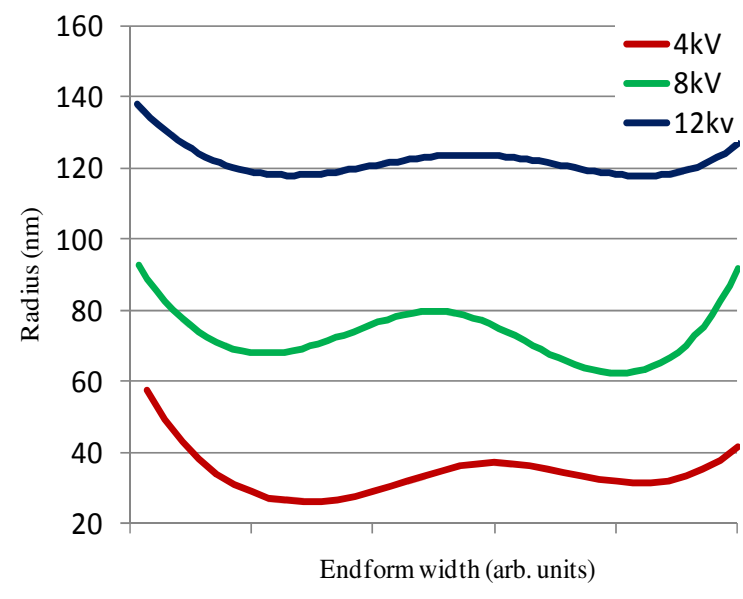

Figure 3
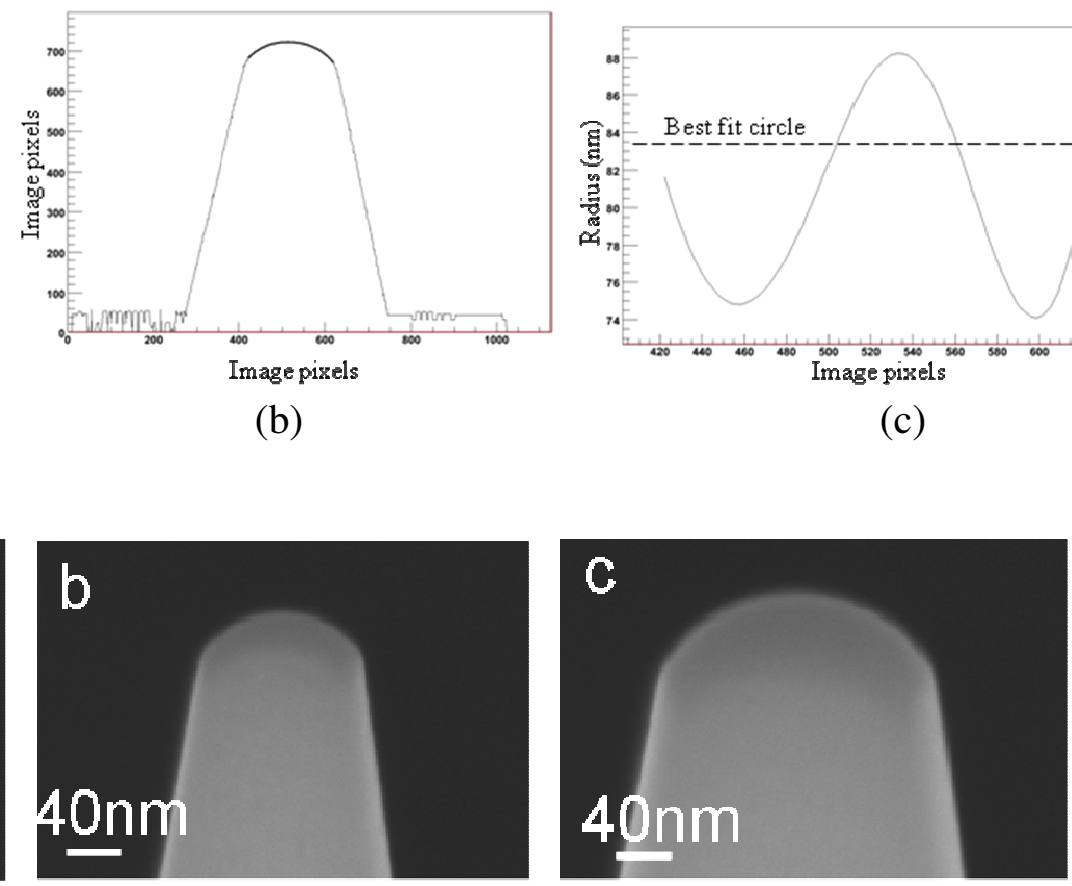

(b)

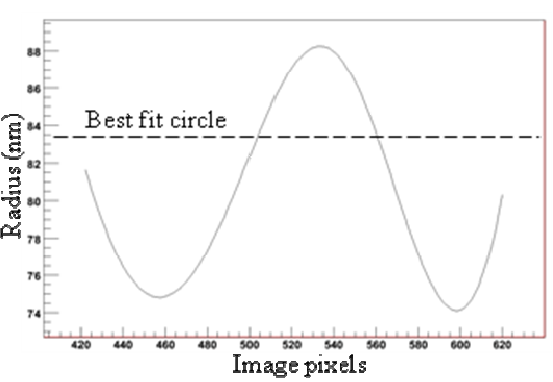

(c)

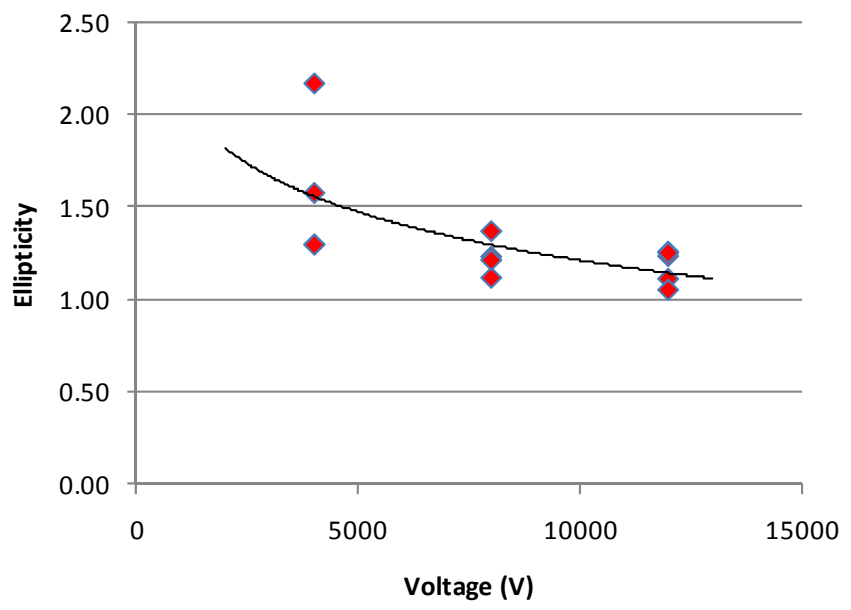

Figure 4

Fig. 1. (a) SEM image of a Si atom probe specimen after analysis by voltage pulsing to $9 \mathrm{kV}$ in a LEAP, (b) edge thresholding used to determine tip outline and (c) local radius variation.

Fig. 2. SEM images, taken perpendicular to the specimen axes, of Si posts having been field evaporated in voltage-pulsed mode in a LEAP to (a) $4 \mathrm{kV}$, (b) $8 \mathrm{kV}$, and (c) $12 \mathrm{kV}$.[6] Fig. 3. Local radius plots of $\mathrm{Si}$ specimens evaporated to 4,8 , and $12 \mathrm{kV}$. The ratio between the maxima and minima decreases with increasing voltage applied to the specimen.

Fig. 4. Variation in ellipticity with increasing voltage. 